\title{
An integrative pan-cancer investigation reveals common genetic and transcriptional alterations of AMPK pathway genes as important predictors of clinical outcomes across major cancer types
}

\author{
Wai Hoong Chang and Alvina G. Lai ${ }^{*}$
}

\begin{abstract}
Background: The AMP-activated protein kinase (AMPK) is an evolutionarily conserved regulator of cellular energy homeostasis. As a nexus for transducing metabolic signals, AMPK cooperates with other energy-sensing pathways to modulate cellular responses to metabolic stressors. With metabolic reprogramming being a hallmark of cancer, the utility of agents targeting AMPK has received continued scrutiny and results have demonstrated conflicting effects of AMPK activation in tumorigenesis. Harnessing multi-omics datasets from human tumors, we seek to evaluate the seemingly pleiotropic, tissue-specific dependencies of AMPK signaling dysregulation.
\end{abstract}

Methods: We interrogated copy number variation and differential transcript expression of 92 AMPK pathway genes across 21 diverse cancers involving over 18,000 patients. Cox proportional hazards regression and receiver operating characteristic analyses were used to evaluate the prognostic significance of AMPK dysregulation on patient outcomes.

Results: A total of 24 and seven AMPK pathway genes were identified as having loss- or gain-of-function features. These genes exhibited tissue-type dependencies, where survival outcomes in glioma patients were most influenced by AMPK inactivation. Cox regression and log-rank tests revealed that the 24-AMPK-gene set could successfully stratify patients into high- and low-risk groups in glioma, sarcoma, breast and stomach cancers. The 24-AMPK-gene set could not only discriminate tumor from non-tumor samples, as confirmed by multidimensional scaling analyses, but is also independent of tumor, node and metastasis staging. AMPK inactivation is accompanied by the activation of multiple oncogenic pathways associated with cell adhesion, calcium signaling and extracellular matrix organization. Anomalous AMPK signaling converged on similar groups of transcriptional targets where a common set of transcription factors were identified to regulate these targets. We also demonstrated crosstalk between procatabolic AMPK signaling and two pro-anabolic pathways, mammalian target of rapamycin and peroxisome proliferator-activated receptors, where they act synergistically to influence tumor progression significantly.

(Continued on next page)

\footnotetext{
*Correspondence: Alvina.Lai@ucl.ac.uk

Institute of Health Informatics, University College London, 222 Euston Road, London NW1 2DA, UK
}

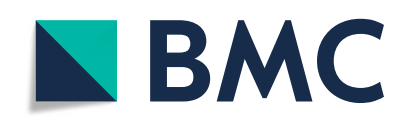

(c) The Author(s). 2020 Open Access This article is licensed under a Creative Commons Attribution 4.0 International License, which permits use, sharing, adaptation, distribution and reproduction in any medium or format, as long as you give appropriate credit to the original author(s) and the source, provide a link to the Creative Commons licence, and indicate if changes were made. The images or other third party material in this article are included in the article's Creative Commons licence, unless indicated otherwise in a credit line to the material. If material is not included in the article's Creative Commons licence and your intended use is not permitted by statutory regulation or exceeds the permitted use, you will need to obtain permission directly from the copyright holder. To view a copy of this licence, visit http://creativecommons.org/licenses/by/4.0/ The Creative Commons Public Domain Dedication waiver (http://creativecommons.org/publicdomain/zero/1.0/) applies to the data made available in this article, unless otherwise stated in a credit line to the data. 
(Continued from previous page)

Conclusion: Genetic and transcriptional aberrations in AMPK signaling have tissue-dependent pro- or anti-tumor impacts. Pan-cancer investigations on molecular changes of this pathway could uncover novel therapeutic targets and support risk stratification of patients in prospective trials.

Keywords: AMPK, Glioma, Loss-of-function, Tumor metabolism, Pan-cancer

\section{Background}

The AMP-activated protein kinase (AMPK) is an evolutionary conserved key player responsible for energy sensing and homeostasis. Orthologous copies of AMPK prevail universally as heterotrimeric complexes where the human genome encodes two genes for the $\alpha$ catalytic subunit, two $\beta$ regulatory subunit genes and three $\gamma$ subunit genes. Historically, AMPK was discovered as a crucial regulator of lipid metabolism [1]. Since then, AMPK is implicated in a wide variety of fundamental metabolic processes as well as in metabolic diseases such as cancer and diabetes [2]. The first link between AMPK and cancer was identified through the tumor-suppressive function of $L K B 1$, which is upstream of the mTOR pathway [3]. The tumor-suppressive roles of AMPK were pharmacologically demonstrated by the application of metabolic inhibitors such as the anti-diabetic metformin and the mimetic of AMP, AICAR [4-6]. Numerous studies have since compellingly established the promiscuous nature of these pharmacological agents, whereby the inhibition of cancer cell proliferation occurs through non-specific AMPK-independent avenues [7, 8].

In contrast to the tumor-suppressive results from pharmacological studies, genetic experiments on cancer cells have credibly demonstrated that AMPK activation is crucial for tumor progression and survival [9-12]. A myriad of metabolic stressors, such as oxygen deprivation, nutrient starvation and oxidative stress, exists within the tumor microenvironment. Metabolic reprogramming during carcinogenesis would thus trigger AMPK activation to enable cells to survive under conditions of stress typically found in the tumor microenvironment, hence conferring an overall tumor-promoting effect. AMPK is also shown to support cancer growth and migration through crosstalk with other pro-oncogenic pathways. For instance, overexpression of oncogenes $M Y C$ and $S R C$ or the loss of the tumor suppressor folliculin could lead to AMPK activation [13-17].

Genetic and pharmacological studies have paved the way for our understanding of the function of AMPK in cancer. However, anti- and pro-neoplastic features of AMPK remain controversial potentially due to the oversimplification of AMPK-modulated processes in in vitro and non-human in-vivo models. The genetic and clinical landscape of AMPK signaling has not been systematically investigated. Thus, our study aims to address an unmet need to rigorously investigate the role of AMPK in diverse cellular context using multi-omics data from actual tumors where we examined somatic copy number alterations, transcriptional and clinical profiles of tumors from 21 cancer types. Our analyses of clinical samples at scale would complement evidence from pharmacological and genetic studies to better elucidate the multi-faceted and cell-specific nature of AMPK signaling on tumor progression.

\section{Methods \\ AMPK pathway genes and cancer cohorts}

Ninety-two AMPK pathway genes were retrieved from the Kyoto encyclopedia of genes and genomes (KEGG) database (Additional file 1). Clinical, genomic and transcriptomic datasets of 21 cancers involving 18,484 patients were downloaded from the Cancer genome atlas (TCGA) [18].

\section{Copy number variation, differential expression, multidimensional scaling and survival analyses}

Detailed methods of the above analyses were previously published and thus will not be repeated here as per the journal guidelines [19-26]. To summarize, discrete amplification and deletion indicators for copy number variation analyses were obtained from GISTIC gene-level tables [27]. GISTIC values of +1 and -1 were annotated as shallow amplification and shallow deletion (heterozygous) events respectively. GISTIC values of +2 and -2 were annotated as deep amplification and deep (homozygous) deletion events respectively. Multidimensional scaling analyses and permutational multivariate analysis of variance (PERMANOVA) were performed using the $R$ vegan package. Survival analyses were performed using Cox proportional hazards regression and the log-rank test. Sensitivity and specificity of the 24-AMPK-gene set were assessed using receiver operating characteristic analyses. Differential expression analyses were performed on patients stratified into high- (4th quartile) and low- (1st quartile) expressing groups using the 24gene-set to determine the transcriptional effects of anomalous AMPK signaling.

\section{Pathway and transcription factor analyses}

Genes that were differentially expressed (DEGs) between the 4th and 1st quartile patient groups were mapped to 
KEGG, Gene Ontology and Reactome databases using g: profiler [28] to ascertain biological processes and signaling pathways that were enriched. The Enrichr tool [29, 30] was used to map DEGs to the ChEA and ENCODE transcription factor (TF) databases to identify TFs that were significantly enriched as regulators of the DEGs.

\section{Calculating the 24-AMPK-gene score, peroxisome proliferator-activated receptors (PPAR) score and mammalian target of rapamycin (mTOR) score}

AMPK scores were calculated from the mean expression of the following genes: $S L C 2 A 4, F O X O 3, P P P 2 C B$, PIK3CD, CAB39L, CCNA1, FBP1, FBP2, FOXO1, HMGC $R$, IRS2, PIK3R1, SIRT1, TBC1D1, PPARGC1A, PPP2R2C, MLYCD, PFKFB3, PPP2R2B, PRKAA2, LEPR, CAB39, IRS1 and PFKFB1. PPAR scores for each patient were calculated by taking the mean expression of PPAR signature genes: PLIN5, PPARG, ACADM, GK, CPT2, SCP2, ACAA1, APOA1, PPARA, ACOX2, ANGPTL4, FABP3, PLIN2, AQP7, ACSL1, FABP5, ACADL, and PCK2 [19]. mTOR/PI3K/AKT scores for each patient were calculated using the following equation: $\mathrm{mTOR} /$ $\mathrm{PI} 3 \mathrm{~K} / \mathrm{AKT}$ score $=A K T+m T O R+G S K 3+S 6 K+S 6-$ PTEN [31].

All figures were generated using $\mathrm{R}$ version 3.6 .3 and Adobe Illustrator version CS6.

\section{Results}

Pan-cancer genomic and transcriptional alterations of AMPK pathway genes

Focusing on the genomic and transcriptomic landscape of 92 genes associated with AMPK signaling retrieved from KEGG across 21 cancer types involving 18,484 patients (Additional file 1), we interrogated somatic copy number alterations (SCNA) and mRNA expression (see Additional file 2 for a flowchart illustrating the study design). To determine the effects of genomic alterations in AMPK pathway genes, we classified genes as having high-level amplifications (gains), low-level amplifications, deep (homozygous) deletions and shallow (heterozygous) deletions. To evaluate pan-cancer patterns of SCNAs, we considered genes that were gained or lost in at least $20 \%$ of samples within a cancer type and in at least onethird of cancer types, i.e., at least seven cancer types. A total of 46 genes were recurrently amplified, while 49 genes were recurrently lost (Fig. 1; Additional file 3). AMPK is the central regulator of cellular energy levels, which controls a number of downstream targets, an example being the nuclear receptor HNF4A. Remarkably, $H N F 4 A$ was found to be the most amplified gene; identified as being recurrently amplified in $>20 \%$ of samples in all 21 cancers (Fig. 1; Additional file 3). This is followed by CFTR (18 cancer types) and four other genes that were amplified in 17 cancer types (ADIPOR2, LEP,
PRKAG2 and RHEB) (Fig. 1; Additional file 3). In contrast, $P P P 2 R 2 A$ was the most deleted gene found in $>20 \%$ of samples across 17 cancers, followed by the deletion of $S L C 2 A 4$ in 16 cancers and five additional genes (FOXO3, PPP2CB, PPP2R2D, PPP2R5C and PPP2R5E) in 15 cancer types (Fig. 1; Additional file 3). Among all cancer types, the highest number of amplified AMPK pathway genes was observed in esophageal carcinoma (ESCA; 44 genes) followed by bladder cancer (BLCA; 42 genes) and lung cancer (41 genes in both lung squamous cell carcinoma [LUSC] and adenocarcinoma [LUAD]) (Fig. 1). Glioma tumors (GBMLGG), in contrast, had only five genes that were recurrently amplified (Fig. 1). In terms of somatic deletions, LUSC and ESCA both had 49 genes deleted while no recurrent deletions were observed in papillary renal cell carcinoma (KIRP) (Fig. 1).

We reasoned that SCNAs associated with transcriptional alterations could be considered as putative gainor loss-of-function events. Differential expression analyses between tumor and non-tumor samples in each cancer revealed that 15 and 39 genes were significantly upregulated and downregulated in at least seven cancer types respectively (Additional file 4). Of these differentially expressed genes, seven and 24 genes were also recurrently amplified and deleted respectively (Venn diagram in Fig. 1). Both gene sets were mutually exclusive, i.e., the genes either had gain-or-function or loss-offunction features, but not both.

\section{Molecular underpinnings of patient survival involving putative loss-of-function AMPK pathway components} We next investigated the impact of transcriptional dysregulation of the putative gain- and loss-of-function genes identified previously on patient survival outcomes across all cancer types. Employing Cox proportional hazards regression, we observed that all 31 genes (seven gain-of-function and 24 loss-of-function genes), were prognostic in at least one cancer type (Fig. 2a). The highest number of prognostic genes was observed in glioma (GBMLGG) tumors (26/31 genes), while none of the 31 genes were significantly associated with overall survival outcomes in ESCA and cholangiocarcinoma (CHOL) (Fig. 2a). Intriguingly, although ESCA had the highest number of SCNAs (Fig. 1), none of the genes harbored prognostic information, suggesting that alterations in AMPK signaling components have minimal roles in driving tumor progression and patient outcomes. FBP1 was significantly associated with overall survival outcomes in 10 cancers while $P P P 2 R 2 C$ and $P P P 2 R 2 B$ in 8 cancers (Fig. 2a). FBP2 is the least prognostic gene in only one cancer type, cervical squamous cell carcinoma and endocervical adenocarcinoma; CESC (Fig. 2a). 

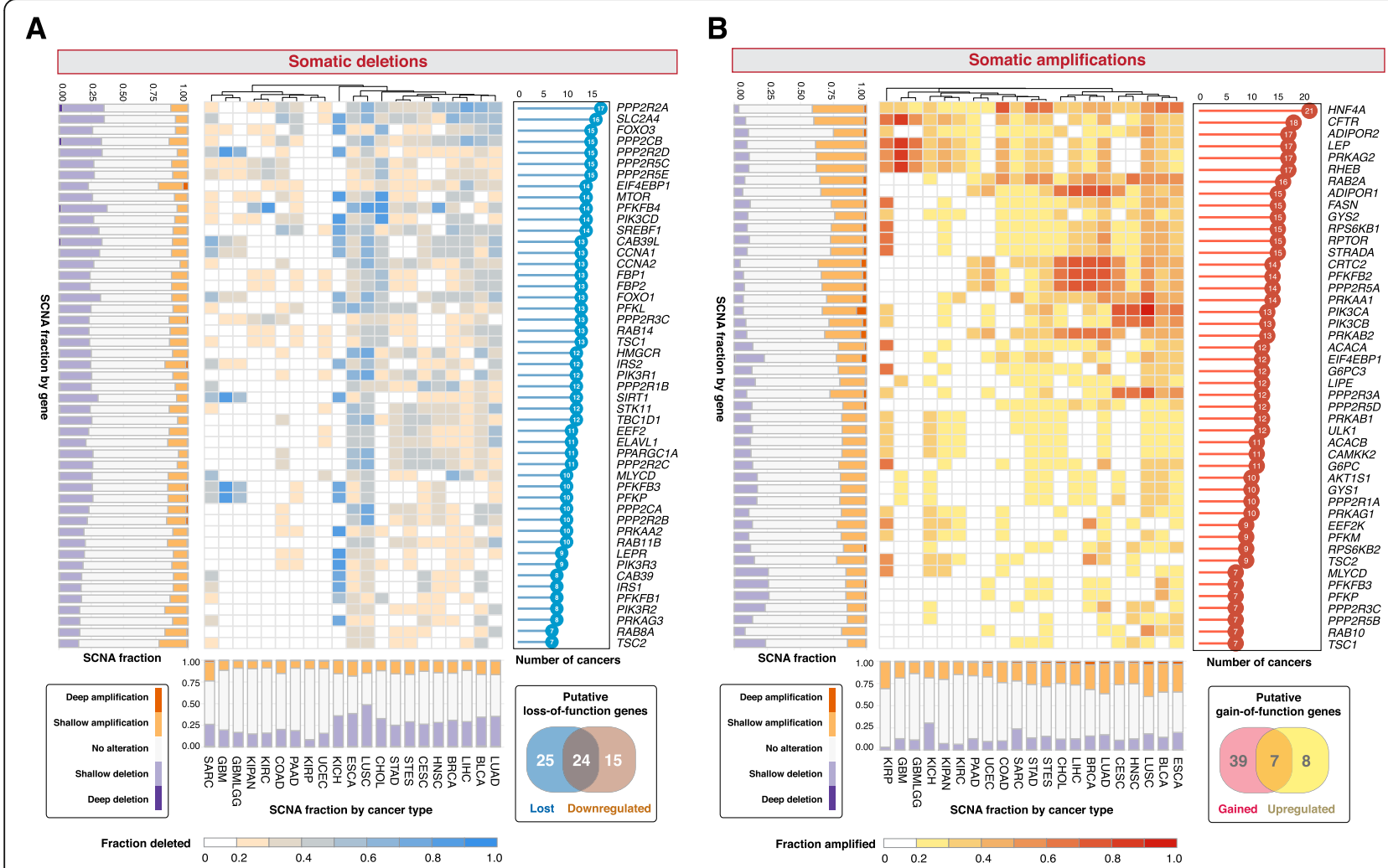

Fig. 1 The landscape of somatic copy number alterations of AMPK pathway genes. Heatmaps depict (a) fraction of samples within each cancer type that harbor somatic deletions and (b) somatic amplifications. Forty-nine genes are recurrently deleted in at least $20 \%$ of tumors within each cancer and in at least seven cancer types. Forty-six genes are recurrently amplified in at least 20\% of tumors within each cancer and in at least seven cancer types. Stacked bar charts on the $y$-axes illustrate the fraction of samples that possess copy number variation of a gene under consideration grouped by shallow and deep deletions or amplifications. Stacked bar charts on the x-axes illustrate the fraction of samples within each cancer type that contain shallow and deep deletions or amplifications. The bar charts on the right of each heatmap depict the number of cancer types with at least $20 \%$ of samples affected by gene deletions and amplifications. The Venn diagrams demonstrate the identification of 24 putative loss- and seven gain-of-function genes from gene sets that are somatically altered and differentially expressed. Cancer cohorts analyzed with corresponding TCGA abbreviations are listed in parentheses: bladder urothelial carcinoma (BLCA), breast invasive carcinoma (BRCA), cervical squamous cell carcinoma and endocervical adenocarcinoma (CESC), cholangiocarcinoma (CHOL), colon adenocarcinoma (COAD), esophageal carcinoma (ESCA), glioblastoma multiforme (GBM), glioma (GBMLGG), head and neck squamous cell carcinoma (HNSC), kidney chromophobe (KICH), pan-kidney cohort (KIPAN), kidney renal clear cell carcinoma (KIRC), kidney renal papillary cell carcinoma (KIRP), liver hepatocellular carcinoma (LIHC), lung adenocarcinoma (LUAD), lung squamous cell carcinoma (LUSC), pancreatic adenocarcinoma (PAAD), sarcoma (SARC), stomach adenocarcinoma (STAD), stomach and esophageal carcinoma (STES) and uterine corpus endometrial carcinoma (UCEC). Number of samples for each cancer type are indicated in parentheses: BLCA (408), BRCA (10939), CESC (304), CHOL (36), COAD (285), ESCA (184), GBM (153), GBMLGG (669), HNSC (520), KICH (66), KIPAN (889), KIRC (533), KIRP (290), LIHC (371), LUAD (515), LUSC (501), PAAD (178), SARC (259), STAD (415), STES (599) and UCEC (370)

Given the prevalence of loss-of-function phenotypes in determining clinical outcomes (Fig. 2a), we proceeded to examine the combined impact of all 24 loss-of-function genes on patient survival and oncogenic dysregulation. To determine the extent of AMPK pathway variation across the 21 cancers, we calculated 'pathway scores' for each of the 18,484 tumor samples by taking the mean transcript expression values of the 24 genes: $S L C 2 A 4$, FOXO3, PPP2CB, PIK3CD, CAB39L, CCNA1, FBP1, FBP2, FOXO1, HMGCR, IRS2, PIK3R1, SIRT1, TBC1D1, PPARGC1A, PPP2R2C, MLYCD, PFKFB3, PPP2R2B, PRKAA2, LEPR, CAB39, IRS1 and PFKFB1. We observed interesting patterns when cancers were ranked from low to high, based on their median pathway scores (Fig. 2b). GBMLGG had the highest median pathway score, while BLCA and CESC were found at the lower end of the spectrum (Fig. 2b). As expected, Kaplan-Meier analysis revealed a significant difference in overall survival between glioma patients $(P<0.0001)$ stratified by low and high 24-gene pathway scores (Fig. 2c). Interestingly, the contribution of AMPK signaling in cancer prognostication is cancer-type dependent. As in glioma, log-rank tests revealed that patients with high 24-gene scores had significantly improved survival outcomes in breast cancer $(P=0,0026)$ and sarcoma $(P=0.021)$ (Fig. $2 c)$. In contrast, high expression of the 24 genes was associated 


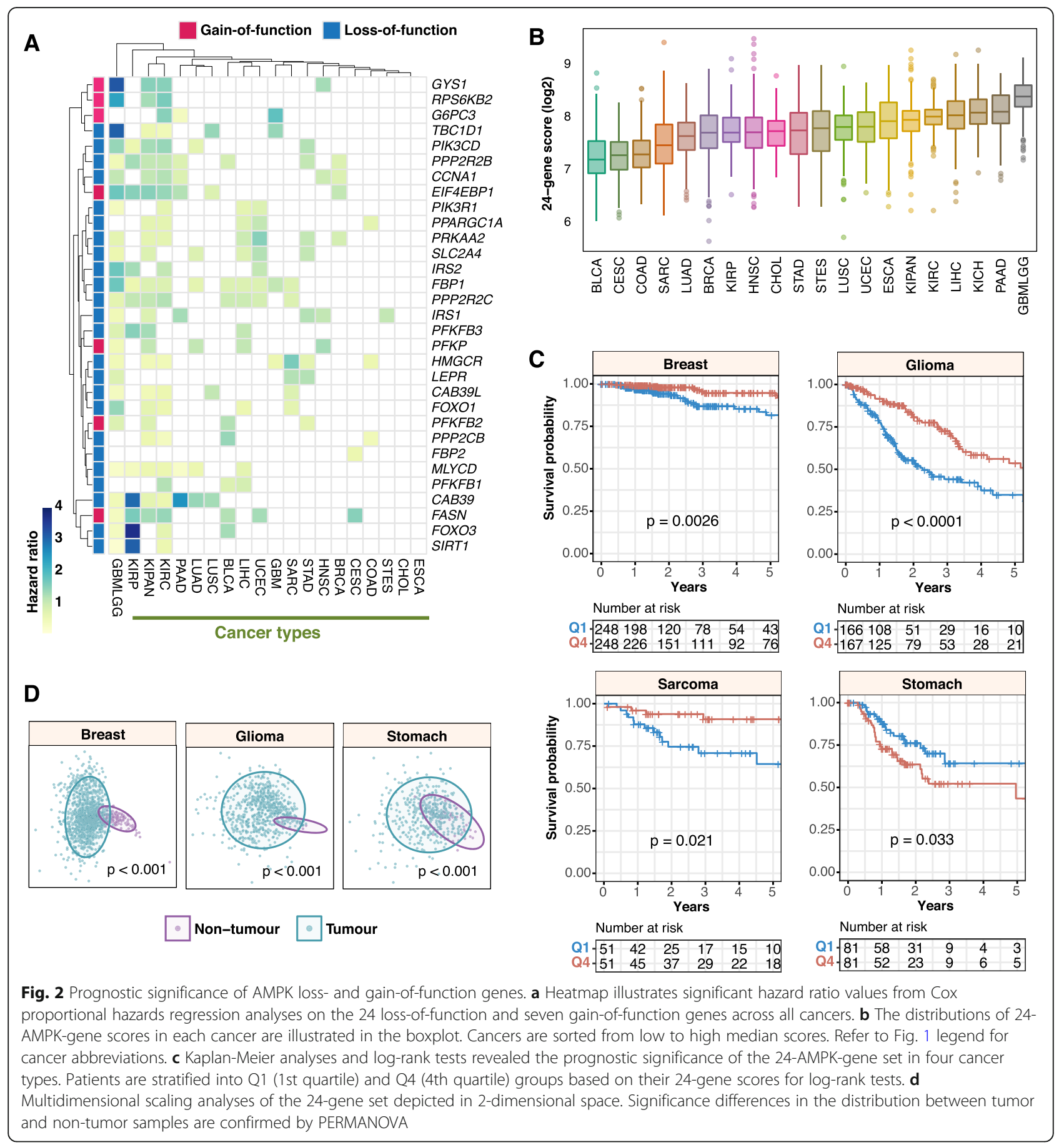

with increased mortality rates in stomach adenocarcinoma $(P=0.033)$ (Fig. 2c). These results were in agreement when independently validated using the Cox regression approach: breast (hazard ratio $[\mathrm{HR}]=0.397$; $P=0.0028)$, glioma $(\mathrm{HR}=0.430 ; P<0.0001)$, sarcoma $(\mathrm{HR}=0.379 ; P=0.021)$ and stomach $(\mathrm{HR}=1.825 ; P=$ 0.034 ) cancers (Additional file 5). Since the 24-gene score could be used to stratify patients into high- and low-risk groups, we predict that when considered together, gene expression values could discriminate tumor from non-tumor samples. Although analysis could not be performed on sarcoma (this dataset only had two non-tumor samples), multidimensional scaling analyses and PERMANOVA tests of breast $(P<0.001)$, glioma $(P<0.001)$ and stomach $(P<0.001)$ cancers revealed significant separation between tumor and non-tumor 
samples in two-dimensional space (Fig. 2d). Overall, this suggests that the 24-gene set could be harnessed as a diagnostic biomarker for early cancer detection.

To determine the independence of the 24-gene set from other clinicopathological features, we employed multivariate Cox regression and observed that the 24gene set is independent of tumor, node and metastasis (TNM) staging (where available) in breast $(\mathrm{HR}=0.403$; $P=0.0043)$ and stomach cancers $(\mathrm{HR}=1.835 ; P=0.038)$ (Additional file 5). Similarly, Kaplan-Meier analyses and log-rank tests confirmed that the 24-gene set allowed further risk stratification of patients with tumors of the same TNM stage: breast $(P<0.0001)$ and stomach $(P=$ 0.022) (Fig. 3a). Furthermore, we observed that within a histological subtype of sarcoma, leiomyosarcoma, patients with elevated AMPK signaling had significantly better survival outcomes $(P=0.0072)$ (Fig. 3a); consistent with our previous observation that high pathway scores were associated with good prognosis in sarcoma (Fig. 2c).

We next explored the predictive performance (sensitivity versus specificity) of the 24-gene set in all four cancer types using receiver operating characteristic analysis. The area under the ROC curve (AUC) is an indication of how well the gene set could predict patient survival, which ranges from 0.5 to 1 . We found that the combined model uniting both 24-gene set and TNM staging outperformed the 24-gene set when considered on its own in breast cancer patients (AUC $=0.749$ vs. 0.699 ) (Fig. 3b). For stomach cancer, the 24-gene set only contributed to a marginally higher AUC when used in combination with TNM staging when compared to the 24-gene set alone (AUC $=0.714$ vs. 0.700). (Fig. 3b). AUCs of the 24-gene set in glioma and sarcoma were

\section{A}
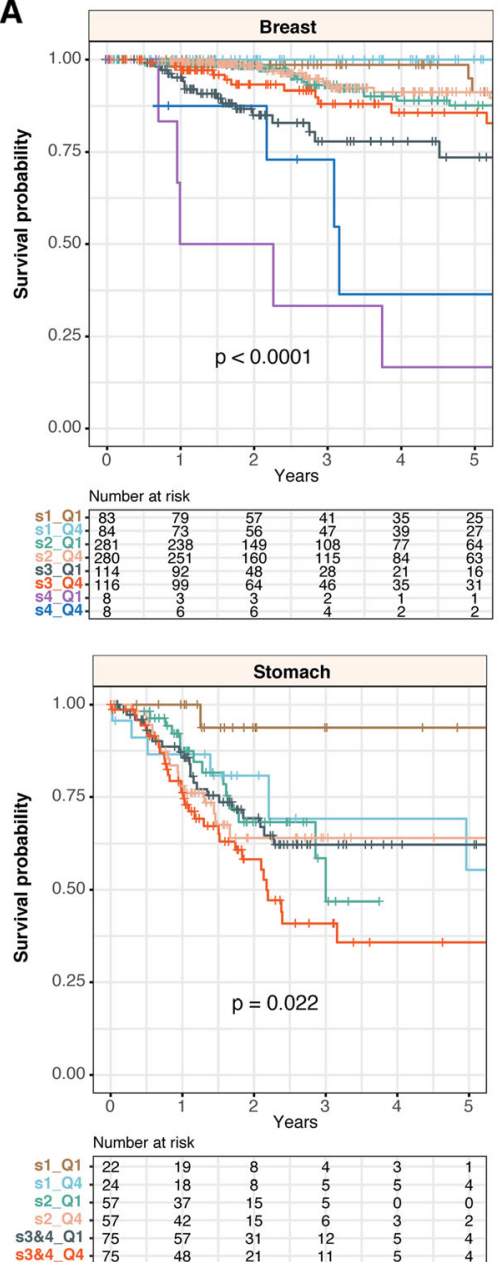

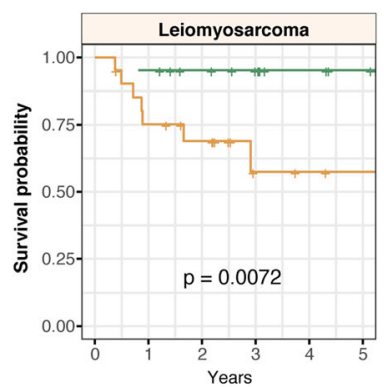

Number at risk

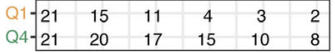

B
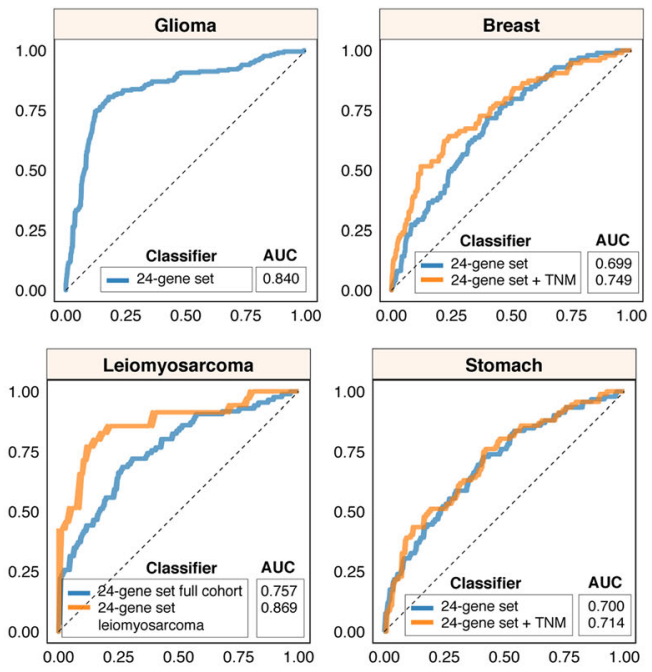

Fig. 3 The 24-AMPK-gene set is independent of tumor stage and histological subtype. a Kaplan-Meier analyses of patients grouped by tumor, node and metastasis (TNM) stage (breast and stomach cancers) or by the histological subtype of leiomyosarcoma and the 24-gene score. For leiomyosarcoma, the log-rank test reveals a significant difference in survival rates between 1st and 4th quartile patients. $\mathbf{b}$ Receiver-operating characteristic (ROC) analyses on the 5-year predictive performance of the 24-gene set. ROC curves generated by the 24-gene set are compared to curves generated from both 24-gene set and TNM staging, where available, or histological subtype. AUC: area under the curve 
0.840 and 0.757 respectively (Fig. 3b). Within the leiomyosarcoma histological subtype, AUC was even higher at 0.869 (Fig. 3b).

\section{Oncogenic transcriptional alterations associated with AMPK pathway inactivation}

AMPK pathway inactivation was associated with altered survival outcomes in patients (Figs. 2 and 3). We predict that this could be due to broad transcriptional dysregulation arising from abnormal AMPK signaling. To investigate this phenomenon, we performed differential expression analyses between patients stratified by the 24-gene set into high (4th quartile) and low (1st quartile) expression groups and found that an outstanding number of 122 common genes that were significantly differentially expressed in all four cancer types (Fig. 4a). The highest number of differentially expressed genes (DEGs) was observed in stomach cancer (2496 genes), followed by sarcoma (1842 genes), glioma (1523 genes) and breast cancer (1086 genes) (Fig. 4a; Additional file 6). The DEGs were mapped to KEGG, Gene Ontology and Reactome databases to determine whether they were associated with any functionally enriched pathways. Intriguingly, all four cancer types share similar patterns of functional enrichments (Fig. 4b and c). For instance, biological processes associated with cell communication, signal transduction, cell differentiation, cell signaling, cell adhesion and cell morphogenesis were enriched in all four cancers (Fig. 4c). In terms of specific signaling pathways, calcium signaling, cAMP signaling, and processes associated with extracellular matrix organization were among the most enriched (Fig. 4c).

To further identify potential transcriptional regulators of the DEGs, we mapped the DEGs to ENCODE and ChEA transcription factor (TF) binding databases. Remarkably, we identified common TFs, shared across all four cancers, that were implicated as direct binding partners of the DEGs (Fig. 4c). Five TFs, SUZ12, SMAD4, REST, EZH2 and NFE2L2, were found to be enriched in all four cancers, suggesting that transcriptional dysregulation of tumors with aberrant AMPK signaling involved direct physical associations of these TFs with target DEGs (Fig. 4c). Curiously, FOXM1 and E2F4 were enriched only in glioma tumors, which deserves further exploration in the next section. Overall, our analyses demonstrated that impaired AMPK signaling resulted in common patterns of oncogenesis, which affect the severity of cancer and consequently, mortality rates in patients.

Downstream targets of EZH2, NFE2L2, REST, SMAD4 and SUZ12 were associated with survival outcomes

Pathways modulating energy homeostatic may transduce signals to influence other cognate signaling modules.
EZH2, NFE2L2, REST, SMAD4 and SUZ12 were all implicated as common transcriptional regulators of DEGs in glioma, sarcoma, breast and stomach cancers, suggesting that altered AMPK signaling converged on similar groups of transcriptional targets. Of all the target DEGs of the aforementioned TFs, 8, 10, 24, 12 and 48 genes were found to be common targets of EZH2, NFE2L2, REST, SMAD4 and SUZ12 respectively in all four cancers (Fig. 5a). Concatenating all five gene sets yielded 71 unique genes, i.e., genes that were binding targets of more than one TF were considered only once. To gain further insights into how AMPK inactivation influences tumor progression, we performed Cox regression analyses to determine the association between each of the 71 genes and survival outcomes. The highest number of prognostic genes was observed in glioma; 66 genes (61 good prognoses and five adverse prognoses) (Fig. 5b). In contrast, 54 out of 71 genes were associated with adverse prognosis in stomach cancer (Fig. 5b). These observations were consistent with the 24-AMPK-gene set being positive and negative prognostic factors in glioma and stomach cancer respectively (Fig. 2), which mirrored the behavior of DEGs identified as a result of aberrant AMPK signaling (Fig. 4c). Of the 71 genes, only 15 and ten were significantly associated with survival outcomes in sarcoma and breast cancer respectively (Fig. 5b). Collectively, our results suggest that the AMPK pathway and its interaction with other signaling modules are key determinants of patient outcomes in multiple cancer types.

\section{Prognostic significance of joint AMPK pathway activity and transcriptional levels of five oncogenic TFs in patients with glioma}

Having discovered the importance of the 24-AMPK gene set, we sought to explore the crosstalk between AMPK signaling and TF activity in glioma. As previously mentioned, glioma had the highest 24-AMPK-gene score (Fig. 2b) with a vast majority of the genes conferring prognostic information (Fig. 2a). Moreover, 66 of the 71 transcriptional targets of the five common TFs identified in patients with altered AMPK signaling were significantly associated with survival outcomes in glioma (Fig. 5b). Additionally, TFs FOXM1 and E2F4 were identified to be enriched only in glioma tumors (Fig. 4c). Thus, we predict that a joint model uniting AMPK and TF expression profiles would allow further delineation of patients into additional risk groups and if so, allowing combined targeting of AMPK and candidate TFs for therapeutic action. As done previously, we calculated AMPK scores for each patient based on the mean expression of the 24 genes. Interestingly, we found that AMPK scores were significantly negatively correlated with $\mathrm{TF}$ expression levels in glioma: E2F4 (rho $=-0.48, P<0.0001$ ), EZH2 


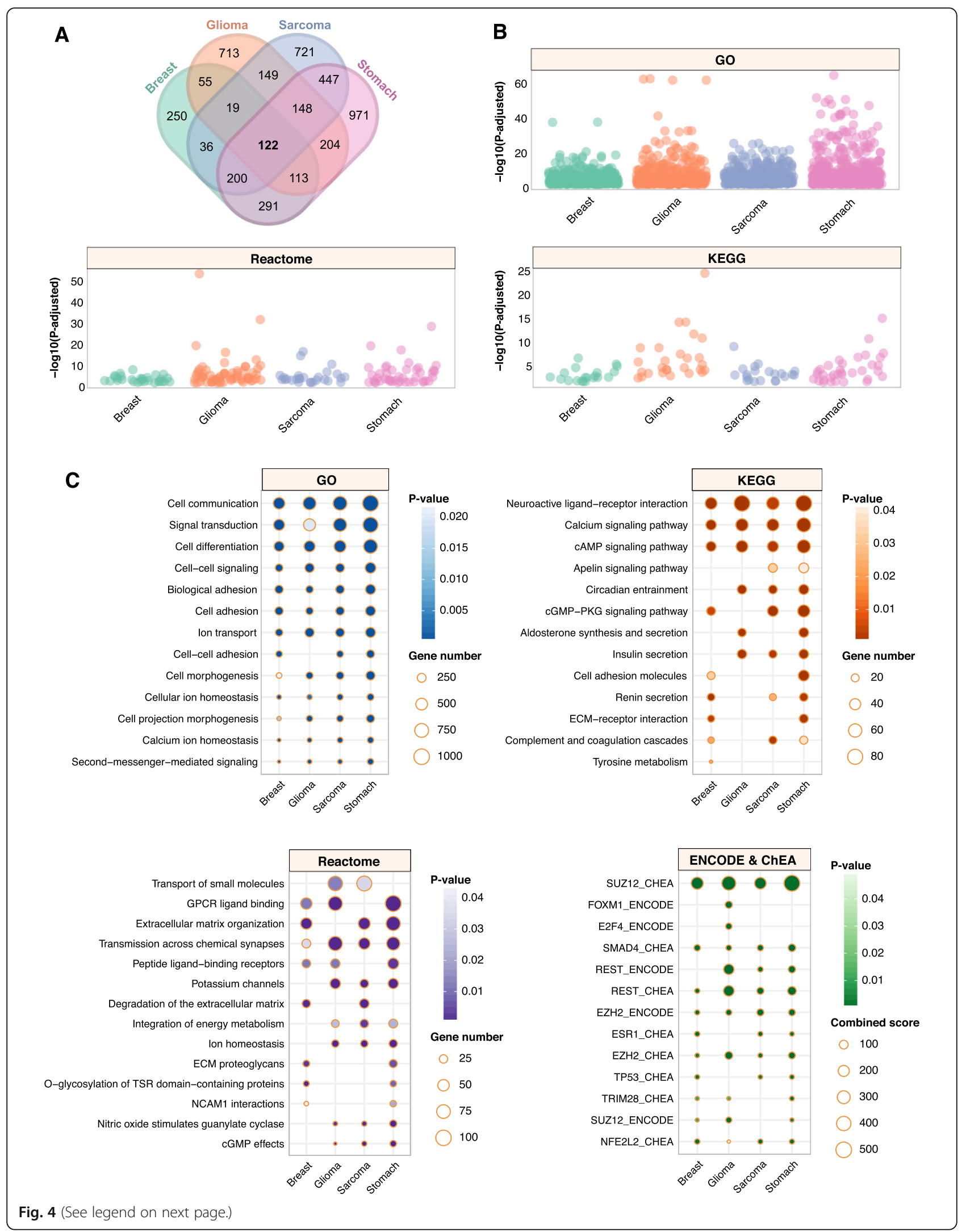


(See figure on previous page.)

Fig. 4 AMPK inactivation drives oncogenic transcriptional alterations in diverse biological processes and signaling modules. a Venn diagram illustrates the number of differentially expressed genes (DEGs) between 1st and 4th quartile patients, as stratified using the 24-AMPK-gene set, in four cancer types. A total of 122 DEGs were common in all four cancers. $\mathbf{b}$ Dot plots depict the number of significantly enriched pathways and biological processes upon the mapping of DEGs to KEGG, Gene Ontology and Reactome databases. Each dot represents an enriched event. c Ontologies that exhibit similar patterns of enrichment across four cancers are shown. DEGs are also mapped to ENCODE and ChEA transcription factor (TF) databases to determine enriched TF binding associated with DEGs

(rho $=-0.57, P<0.0001)$, FOXM1 (rho $=-0.49, P<$ $0.0001)$, SMAD4 (rho $=-0.18, P<0.0001$ ) and SUZ12 (rho $=-0.23, P<0.0001$ ) (Fig. 6a). We subsequently categorized patients into four groups using the median cutoff of the AMPK scores and TF expression values: 1) low-low, 2) high-high, 3) low AMPK score and high TF expression and 4) high AMPK score and low TF expression. Log-rank tests revealed that patients stratified into the four groups had survival rates that were significantly different: E2F4 $(P<0.0001), E Z H 2(P<0.0001)$, FOXM1 $(P<0.0001)$, SMAD4 $(P<0.0001)$ and SUZ12 $(P<$ 0.0001) (Fig. 6b). For E2F4, EZH2, FOXM1 and SUZ12, patients with low AMPK scores and high TF expression performed the worst: E2F4 $(\mathrm{HR}=3.916 ; P<0.0001)$, EZH2 (HR = 4.004; $P<0.0001)$, FOXM1 $(\mathrm{HR}=5.268$; $P<0.0001)$ and SUZ12 $(\mathrm{HR}=2.197 ; P<0.0001)$ (Fig. 6c). For $S M A D 4$, patients within the low-low category had the highest mortality rates $(\mathrm{HR}=3.326 ; P<0.0001)$ (Fig. 6c).

\section{Crosstalk between AMPK and other anabolic-related pathways, PPAR and mTOR}

AMPK's anti-anabolic and pro-catabolic activities may work in concert with other metabolic pathways. To investigate the synergistic effects of AMPK and two proanabolic pathways, peroxisome proliferator-activated receptors (PPAR) and mammalian target of rapamycin (mTOR) signaling in tumor progression, we calculated PPAR and mTOR pathway scores (detailed in the methods section) for each glioma tumor. Low AMPK scores were associated with poor outcomes in glioma (Fig. 2). To evaluate AMPK and PPAR or mTOR as combined models, patients were separated into four groups using the median cutoff, as mentioned previously. Interestingly, when AMPK and PPAR scores were collectively used for patient stratification, we found that patients with low AMPK and high PPAR scores had the highest death rates $(\mathrm{HR}=11.308, P<0.0001)$, confirming that PPAR hyperactivation is associated with poor outcomes in glioma tumors with low AMPK activity [19] (Fig. 7). In contrast, when considering mTOR activity, patients with low AMPK and low mTOR scores performed the worst $(\mathrm{HR}=3.023, P<0.0001)$ (Fig. 7). The results overall suggest that the AMPK pathway could act synergistically with PPAR and mTOR signaling to influence cancer progression significantly.

\section{Discussion}

While the role of AMPK in energy-sensing is well understood, its full potential in metabolic diseases such as cancer remains an open topic of debate. Despite extensive efforts spent on elucidating the role of AMPK signaling $[2,9,11]$, there remains no consensus on whether AMPK promotes or suppresses tumor progression. Exploiting a rich reservoir of pan-cancer datasets afforded to us by TCGA, we performed a thorough examination of genomic and transcriptomic profiles of 92 AMPK pathway genes in diverse cancer types. Our current understanding of AMPK signaling is fueled by genetic studies in cell lines and animal models [2]. Although useful in determining causal relationships, results from in vitro cell lines and animal models may have limited translational relevance as they do not accurately reflect human pathology [32]. Animal models may offer additional mechanistic insights, but limitations in ethics and costs remain. Moreover, the complexity of human cancers is not accurately modeled in animals; less than $8 \%$ of results from animal models are translated to clinical trials [33]. Despite analyses on tumor genetic datasets providing mostly correlative outcomes, they remain valuable in understanding disease-specific molecular pathology when interrogated at scale on large patient groups [34-37], and when results are considered in relation to those obtained from cell lines and animal models.

Employing pan-cancer population data, our study identified conserved and unique patterns of AMPK signaling across diverse cancer types. Analyses at two molecular levels (genetic and transcriptional) yielded a more comprehensive depiction of AMPK signaling, where we identified genes that were both somatically altered and differentially expressed. These putative lossor gain-of-function genes are more likely to impact tumor progression as they are altered at both macromolecular levels. As reported in other studies, we confirmed that AMPK signaling could either be oncogenic or tumor suppressive depending on the cellular context. Intuitively, since AMPK is anti-anabolic, its function may not be fitting for tumor growth and proliferation. This is consistent with reports demonstrating AMPK's tumor suppressive activity [38, 39]. A study on lymphoma demonstrates that AMPK downregulation induces the Warburg effect and hypoxia signaling in mice [40]. 
A
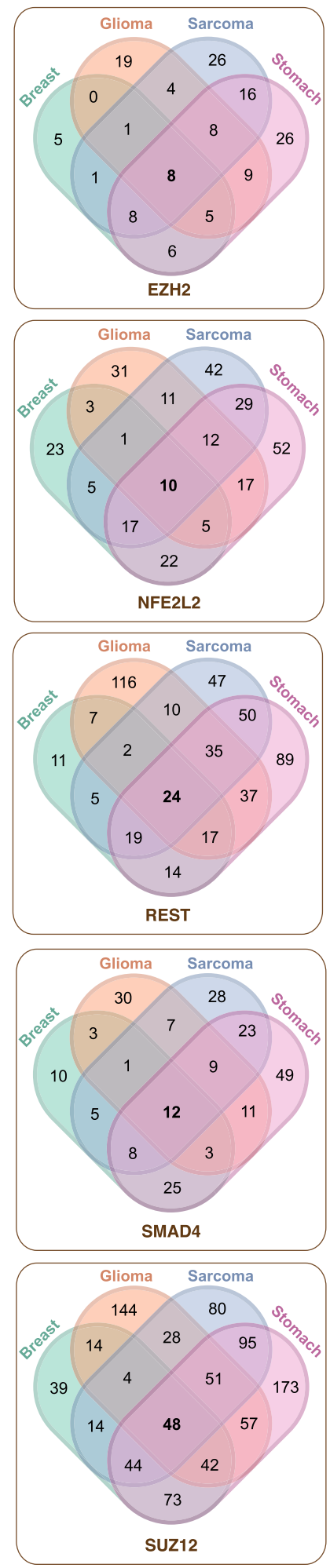

B
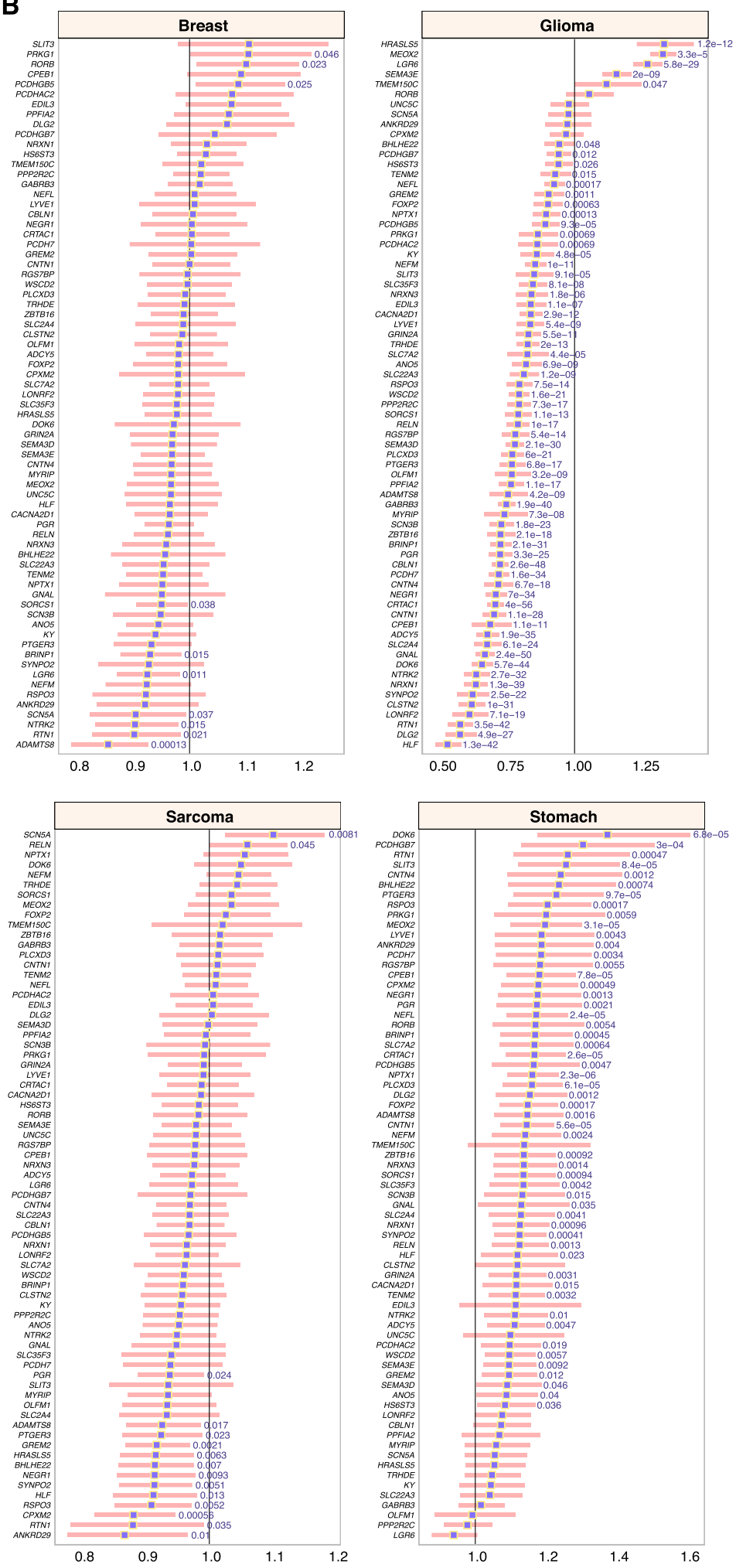

Fig. 5 (See legend on next page.) 
(See figure on previous page.)

Fig. 5 Prognostic significance of DEGs targeted by enriched TFs. a Venn diagrams illustrate the extent of overlap between DEGs targeted by EZH2, NFE2L2, REST, SMAD4 and SUZ12 across four cancers. b Forest plots depict DEGs that are significantly associated with overall survival outcomes. Hazard ratios are denoted as purple squares while pink bars represent the $95 \%$ confidence intervals. Significant Wald test $P$ values are indicated in blue

AMPK is proposed to act as a metabolic gatekeeper to limit cancer cell division; hence, its loss of function would contribute to tumor aggression because of the loss in metabolic checkpoints [40, 41]. AMPK regulates the tumor-suppressive function of the serine/threonine kinase $L K B 1$. Ablation of $L K B 1$ results in enhanced risk of developing gastrointestinal, lung and skin squamous cell cancers $[42,43]$. Moreover, AMPK is shown to inhibit PI3K/AKT/mTOR signaling, which is activated in many cancers $[39,44]$. Also, metabolic inhibitors such as metformin, which indirectly activates AMPK could suppress tumor growth via autophagy induction and mTOR inhibition [39, 45]. Metformin is shown to inhibit the proliferation of estrogen receptor $\alpha(E R \alpha)$ negative and positive breast cancer cell lines through AMPK stimulation [46]. However, when tested in mice models, metformin contributes to enhanced tumor progression and increased angiogenesis, providing us with a glimpse of potential pro-neoplastic effects of AMPK activation [46].

In our study, we observed that high levels of AMPK pathway activity were associated with better outcomes in glioma, breast cancer and sarcoma (Fig. 2); corroborating previous results on the tumor-suppressive function of AMPK. Conversely, the opposite is true in stomach

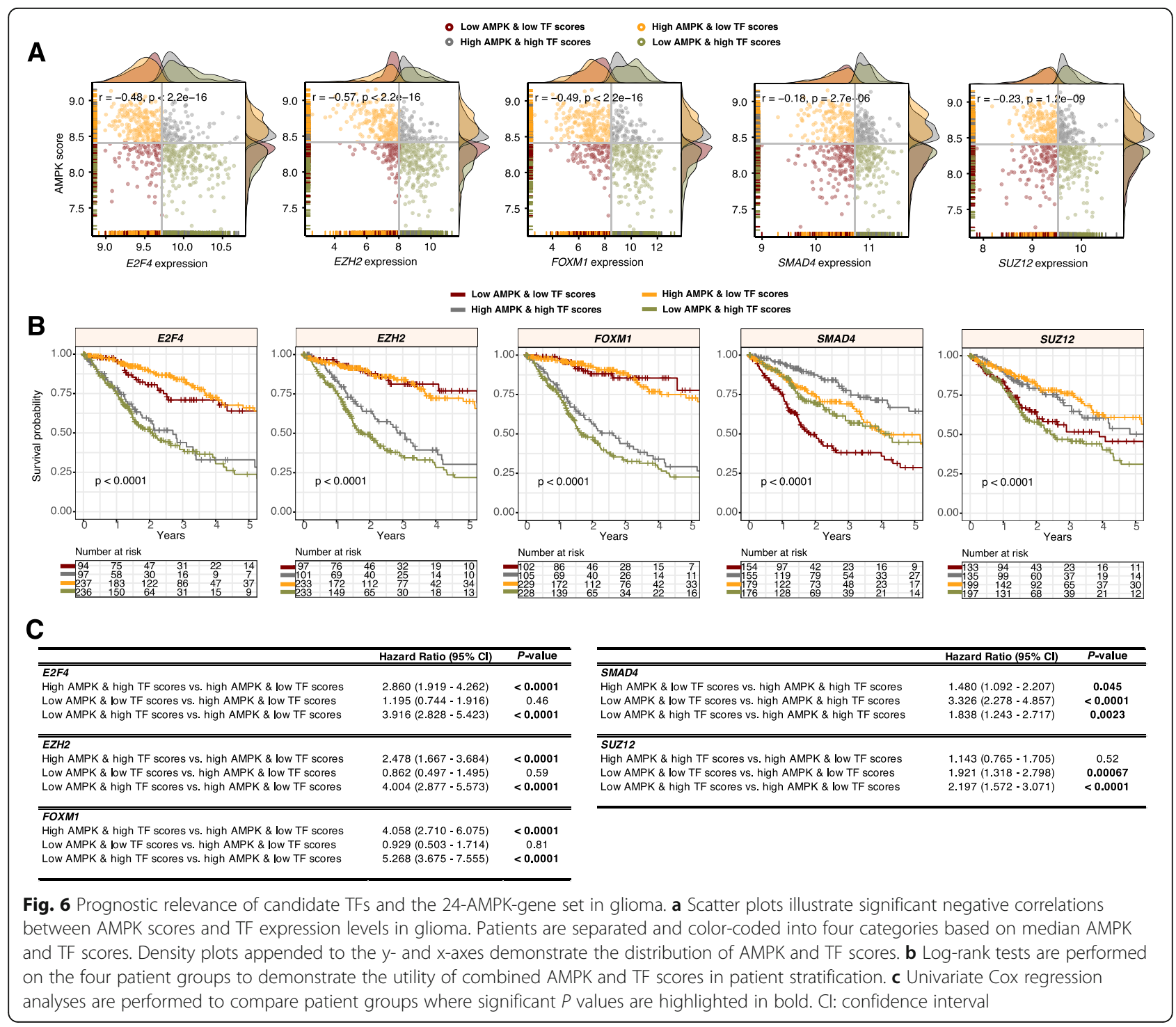



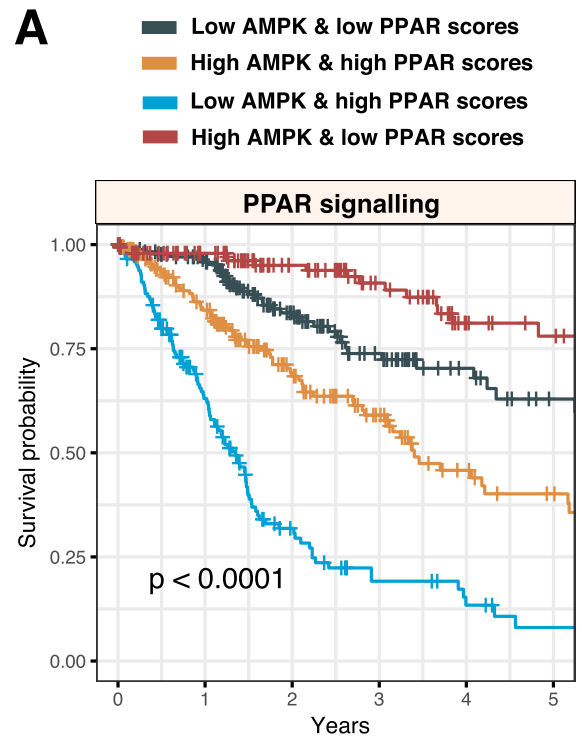

Number at risk

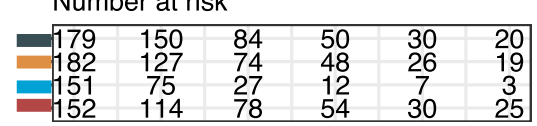

High AMPK \& high mTOR scores

High AMPK \& low mTOR scores

Low AMPK \& low mTOR scores

- Low AMPK \& high mTOR scores

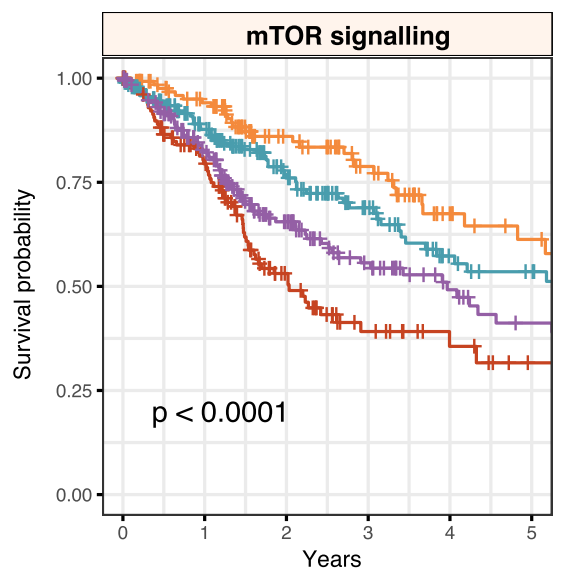

Number at risk

\begin{tabular}{|r|r|r|r|r|r|}
\hline 138 & 106 & 67 & 48 & 25 & 19 \\
196 & 135 & 85 & 54 & 31 & 25 \\
135 & 88 & 39 & 18 & 10 & 4 \\
195 & 137 & 72 & 44 & 27 & 19 \\
\hline
\end{tabular}

B

\begin{tabular}{lcc}
\hline & Hazard Ratio (95\% Cl) & P-value \\
\hline \hline PPAR signalling & & \\
High AMPK \& high PPAR scores vs. high AMPK \& low PPAR scores & $3.911(2.430-6.294)$ & $<\mathbf{0 . 0 0 0 1}$ \\
Low AMPK \& high PPAR scores vs. high AMPK \& low PPAR scores & $11.308(7.075-18.074)$ & $<\mathbf{0 . 0 0 0 1}$ \\
Low AMPK \& low PPAR scores vs. high AMPK \& low PPAR scores & $2.070(1.241-3.452)$ & $\mathbf{0 . 0 0 5 3}$ \\
& & \\
\hline mTOR signalling & $1.381(0.918-2.079)$ & 0.12 \\
High AMPK \& low mTOR scores vs. high AMPK \& high mTOR scores & $3.023(1.997-4.571)$ & $<\mathbf{0 . 0 0 0 1}$ \\
Low AMPK \& low mTOR scores vs. high AMPK \& high mTOR scores & $\mathbf{0 . 0 0 0 2 6}$ \\
\hline Low AMPK \& high mTOR scores vs. high AMPK \& high mTOR scores & $2.071(1.402-3.060)$ & \\
\hline
\end{tabular}

Fig. 7 Crosstalk between AMPK signaling and PPAR or mTOR pathways in glioma. a Log-rank tests are performed on patient groups separated into four categories based on median AMPK and PPAR or mTOR scores. $\mathbf{b}$ Univariate Cox regression analyses are performed to compare patient groups where significant $P$ values are highlighted in bold. Cl: confidence interval

cancer, where AMPK activation contributes to adverse outcomes (Fig. 2). It has now been increasingly clear that AMPK activation can also be pro-tumorigenic [10]. Double knockout of AMPK $\alpha 1$ and AMPK $\alpha 2$ in mouse embryonic fibroblasts result in impaired tumor formation [47]. AMPK knockdown in pancreatic cancer cells impairs anchorage-dependent growth and reduces cell viability under glucose deprived conditions [48]. AMPK signaling induces cell migration in prostate cancer cells [49] while AMPK knockdown inhibits cell proliferation and promotes apoptosis [50]. In liver cancer cells retrieved from primary mouse tumors, AMPK activity is required for Myc-driven carcinogenesis [16]. Taken together, these studies suggest that AMPK activation due to metabolic stress within the tumor microenvironment is crucial for the survival of cancerous cells.
Although 19 of the 21 cancers had at least one gainof-function or loss-of-function gene that correlated with survival outcomes, glioma tumors were most influenced (Fig. 2a). The consequence of dysregulated AMPK signaling was further explored in glioma, where the 24AMPK-gene set and each of the five TFs (identified as regulators of AMPK-associated DEGs) were considered jointly for patient stratification. We observed oncogenic roles of E2F4, EZH2, FOXMI and SUZ12 - patients with high expression of these TFs had higher mortality rates (Fig. 6b). Since the 24-AMPK-gene set was a positive prognostic factor in glioma where high expression of the genes was associated with better outcomes (Fig. 2c), glioma patients harboring low AMPK scores and high oncogenic TF scores performed the worst. Our results are confirmed by other reports on the crosstalk between 
AMPK signaling and E2F4, EZH2, FOXMI or SUZ12 and their effects on oncogenic progression [51-54]. Our analyses on SMAD4 in glioma revealed a likely tumorsuppressive role of the gene (Fig. 6b), which is corroborated by another study demonstrating reduced SMAD4 expression during glioma tumor progression [55]. When merged with the anti-neoplastic effects of AMPK activation, 5-year survival rates were improved by almost $30 \%$ compared to individuals within the low-low category (Fig. 6b). SMAD4 protein expression is lost in gastric cancer cells and loss of expression in primary gastric adenocarcinomas are associated with poor survival [56]. SMAD4 is also commonly inactivated in gastrointestinal cancers $[57,58]$. Restoration of SMAD4 expression in pancreatic cancer cells inhibits tumor function in vivo by influencing angiogenesis through decreased VEGF expression [59].

\section{Conclusion}

Our study has demonstrated that there is far from a single unifying role of AMPK signaling in cancer progression. Harnessing multiplatform datasets, this study provides a comprehensive depiction of how AMPK signaling is manifested in a variety of cancers. We anticipate that this repertoire of organized data would be explored by the research community to devise additional research plans aiming to better understand the roles of AMPK in cancer development. We demonstrated that the pro- or anti-neoplastic effects of AMPK activation is cancer-type dependent. Targeting AMPK for treating metabolic diseases such as diabetes has been well established. Also, the potential for targeting AMPK in cancer therapy has been elegantly reviewed [8]. However, since AMPK activation is a double-edged sword, careful considerations need to be in place before AMPK can be viably deployed in clinical settings. Our study provides a comprehensive catalog of clinically actionable genetic variations which could be used for patient stratification in prospective clinical trials testing the capabilities of AMPK antagonists or agonists as potential treatments for cancer.

\section{Supplementary information}

Supplementary information accompanies this paper at https://doi.org/10. 1186/s12885-020-07286-2.

\footnotetext{
Additional file 1. List of 92 AMPK pathway genes.

Additional file 2. Flowchart depicting the study design.

Additional file 3. Somatic copy number alterations of AMPK pathway genes above $20 \%$ of samples within each cancer type.

Additional file 4 Differential transcript expression of AMPK pathway genes. Differential expression analyses performed on tumor vs. nontumor samples reveal (A) 39 significantly downregulated genes and (B) 15 significantly upregulated genes. Only genes that are differentially expressed in at least seven cancer types are shown. The bar charts on
}

the right of each heatmap depict the number of cancers affected by altered transcript expression.

Additional file 5. Univariate and multivariate Cox proportional hazards regression analyses.

Additional file 6. Differentially expressed genes between patients separated by the 24-AMPK-gene set into 4th and 1st quartiles.

\section{Abbreviations}

AMPK: AMP-activated protein kinase; KEGG: Kyoto Encyclopedia of Genes and Genomes; PERMANOVA: Permutational multivariate analysis of variance; DEGs: Differentially expressed genes; SCNA: Somatic copy number alterations; HR: Hazard ratio; TNM: Tumor, node and metastasis; ROC: Receiver operating characteristic; AUC: Area under the curve; TF: Transcription factor; Cl: Confidence interval; PPAR: Peroxisome proliferator-activated receptors; mTOR: Mammalian target of rapamycin; TCGA: The Cancer Genome Atlas

\section{Acknowledgments}

The results shown here are based upon data generated by The Cancer Genome Atlas Research Network: https://www.cancer.gov/tcga.

\section{Authors' contributions}

$A G L$ designed the study and supervised the research. WHC and AGL analyzed the data, interpreted the results and wrote the initial manuscript draft. AGL revised the manuscript draft. All authors approved the final submitted manuscript.

\section{Funding \\ None.}

\section{Availability of data and materials}

The datasets supporting the conclusions of this article are included within the article and its additional files.

Ethics approval and consent to participate

No permissions were needed to use data from TCGA as it is publicly available.

\section{Consent for publication}

Not applicable.

\section{Competing interests}

None.

Received: 29 March 2020 Accepted: 10 August 2020

Published online: 17 August 2020

\section{References}

1. Carling D, Zammit VA, Hardie DG. A common bicyclic protein kinase cascade inactivates the regulatory enzymes of fatty acid and cholesterol biosynthesis. FEBS Lett. 1987;223:217-22 Wiley Online Library.

2. Dasgupta B, Chhipa RR. Evolving lessons on the complex role of AMPK in normal physiology and cancer. Trends Pharmacol Sci. 2016;37:192-206 Elsevier.

3. Hawley SA, Boudeau J, Reid JL, Mustard KJ, Udd L, Mäkelä TP, et al. Complexes between the LKB1 tumor suppressor, STRADa $/ \beta$ and MO25a/ $\beta$ are upstream kinases in the AMP-activated protein kinase cascade. J Biol. 2003;2:28 BioMed Central.

4. Guo D, Hildebrandt IJ, Prins RM, Soto H, Mazzotta MM, Dang J, et al. The AMPK agonist AICAR inhibits the growth of EGFRvlll-expressing glioblastomas by inhibiting lipogenesis. Proc Natl Acad Sci. 2009;106: 12932-7.

5. Dowling RJO, Zakikhani M, Fantus IG, Pollak M, Sonenberg N. Metformin inhibits mammalian target of rapamycin-dependent translation initiation in breast cancer cells. Cancer Res. 2007;67:10804-12 AACR.

6. Guigas B, Detaille D, Chauvin C, Batandier C, De Oliveira F, Fontaine E, et al. Metformin inhibits mitochondrial permeability transition and cell death: a pharmacological in vitro study. Biochem J. 2004;382:877-84 Portland Press Limited. 
7. Santidrián AF, González-Gironès DM, Iglesias-Serret D, Coll-Mulet L, Cosialls AM, de Frias $M$, et al. AICAR induces apoptosis independently of AMPK and p53 through up-regulation of the BH3-only proteins BIM and NOXA in chronic lymphocytic leukemia cells. Blood Am Soc Hematol. 2010;116:3023-32.

8. Li W, Saud SM, Young MR, Chen G, Hua B. Targeting AMPK for cancer prevention and treatment. Oncotarget. 2015;6:7365 Impact Journals, LLC.

9. Jeon S-M, Hay N. The dark face of AMPK as an essential tumor promoter. Cell Logist. 2012;2:197-202 Taylor \& Francis.

10. Faubert B, Vincent EE, Poffenberger MC, Jones RG. The AMP-activated protein kinase (AMPK) and cancer: many faces of a metabolic regulator. Cancer Lett. 2015;356:165-70 Elsevier.

11. Liang J, Mills GB. AMPK: a contextual oncogene or tumor suppressor? Cancer Res. 2013;73:2929-35 AACR.

12. Hardie DG, Alessi DR. LKB1 and AMPK and the cancer-metabolism link-ten years after. BMC Biol. 2013;11:36 BioMed Central.

13. Possik E, Jalali Z, Nouët Y, Yan M, Gingras M-C, Schmeisser K, et al. Folliculin regulates ampk-dependent autophagy and metabolic stress survival. PLoS Genet. 2014;10:e1004273 Public Library of Science.

14. Yan M, Gingras M-C, Dunlop EA, Nouët Y, Dupuy F, Jalali Z, et al. The tumor suppressor folliculin regulates AMPK-dependent metabolic transformation. J Clin Invest. 2014;124:2640-50 Am Soc Clin Investig.

15. Mizrachy-Schwartz S, Cohen N, Klein S, Kravchenko-Balasha N, Levitzki A. Up-regulation of AMP-activated protein kinase in cancer cell lines is mediated through c-Src activation. J Biol Chem. 2011;286:15268-77 ASBMB.

16. Liu L, Ulbrich J, Müller J, Wüstefeld T, Aeberhard L, Kress TR, et al. Deregulated MYC expression induces dependence upon AMPK-related kinase 5. Nature. 2012;483:608 Nature Publishing Group.

17. Ríos M, Foretz M, Viollet B, Prieto A, Fraga M, Costoya JA, et al. AMPK activation by oncogenesis is required to maintain cancer cell proliferation in astrocytic tumors. Cancer Res. 2013;73:2628-38 AACR.

18. Weinstein JN, Collisson EA, Mills GB, Shaw KRM, Ozenberger BA, Ellrott $K$, et al. The cancer genome atlas pan-cancer analysis project. Nat Genet. 2013; 45:1113 Nature Publishing Group.

19. Chang WH, Lai AG. The pan-cancer mutational landscape of the PPAR pathway reveals universal patterns of dysregulated metabolism and interactions with tumor immunity and hypoxia. Ann N Y Acad Sci. 2019; 1448(1):65-82. https://doi.org/10.1111/nyas.14170.

20. Chang WH, Forde $D$, Lai AG. A novel signature derived from immunoregulatory and hypoxia genes predicts prognosis in liver and five other cancers. J Transl Med. 2019;17:14.

21. Chang WH, Forde D, Lai AG. Dual prognostic role of 2-oxoglutaratedependent oxygenases in ten cancer types: implications for cell cycle regulation and cell adhesion maintenance. Cancer Commun. 2019;39(1):23. https://doi.org/10.1186/s40880-019-0369-5.

22. Chang WH, Lai AG. Timing gone awry: distinct tumour suppressive and oncogenic roles of the circadian clock and crosstalk with hypoxia signalling in diverse malignancies. J Transl Med. 2019;17(1):132. https://doi.org/10. 1186/s12967-019-1880-9.

23. Chang WH, Lai AG. Transcriptional landscape of DNA repair genes underpins a pan-cancer prognostic signature associated with cell cycle dysregulation and tumor hypoxia. DNA Repair (Amst). 2019;78:142-53 Elsevier. Available from: http://www.ncbinlm.nih.gov/pubmed/31054516.

24. Chang WH, Lai AG. Pan-cancer genomic amplifications underlie a WNT hyperactivation phenotype associated with stem cell-like features leading to poor prognosis. Transl Res. 2019;208:47-62. https://doi.org/10.1016/j.trsl. 2019.02.008

25. Chang WH, Lai AG. Aberrations in Notch-Hedgehog signalling reveal cancer stem cells harbouring conserved oncogenic properties associated with hypoxia and immunoevasion. Br J Cancer. 2019; 10.1038/s4

26. Chang WH, Lai AG. An immunoevasive strategy through clinically-relevant pan-cancer genomic and transcriptomic alterations of JAK-STAT signaling components. Mol Med. 2019;25:1-14.

27. Mermel CH, Schumacher SE, Hill B, Meyerson ML, Beroukhim R, Getz G. GIST IC2. 0 facilitates sensitive and confident localization of the targets of focal somatic copy-number alteration in human cancers. Genome Biol. 2011;12: R41 BioMed Central.

28. Reimand J, Kull M, Peterson H, Hansen J, Vilo J. g: Profiler-a web-based toolset for functional profiling of gene lists from large-scale experiments. Nucleic Acids Res. 2007;35:W193-200 Oxford University Press.
29. Kuleshov MV, Jones MR, Rouillard AD, Fernandez NF, Duan Q, Wang Z, et al. Enrichr: a comprehensive gene set enrichment analysis web server 2016 update. Nucleic Acids Res. 2016;44:W90-7 Oxford University Press.

30. Chen EY, Tan CM, Kou Y, Duan Q, Wang Z, Meirelles GV, et al. Enrichr: interactive and collaborative HTML5 gene list enrichment analysis tool. BMC Bioinformatics. 2013;14:128 BioMed Central.

31. Creighton CJ, Fu X, Hennessy BT, Casa AJ, Zhang Y, Gonzalez-Angulo AM, et al. Proteomic and transcriptomic profiling reveals a link between the PI3K pathway and lower estrogen-receptor (ER) levels and activity in ER+ breast cancer. Breast Cancer Res. 2010;12:R40 BioMed Central.

32. Guo S, Qian W, Cai J, Zhang L, Wery JP, Li QX. Molecular pathology of patient tumors, patient-derived xenografts, and cancer cell lines. Cancer Res. 2016;76:4619-26.

33. Mak IWY, Evaniew N, Ghert M. Lost in translation: animal models and clinical trials in cancer treatment. Am J Transl Res. 2014;6:114 e-Century Publishing Corporation.

34. Korkut A, Zaidi S, Kanchi RS, Rao S, Gough NR, Schultz A, et al. A pan-Cancer analysis reveals high-frequency genetic alterations in mediators of signaling by the TGF- $\beta$ superfamily. Cell Syst. 2018;7:422-37.

35. Liu J, Lichtenberg T, Hoadley KA, Poisson LM, Lazar AJ, Cherniack AD, et al. An Integrated TCGA Pan-Cancer Clinical Data Resource to Drive HighQuality Survival Outcome Analytics. Cell. 2018;173:400-416.e11.

36. Thorsson V, Gibbs DL, Brown SD, Wolf D, Bortone DS, Ou Yang TH, et al. The Immune Landscape of Cancer. Immunity. 2018;48:812-830.e14.

37. Ge Z, Leighton JS, Wang Y, Peng $X$, Chen Z, Chen H, et al. Integrated Genomic Analysis of the Ubiquitin Pathway across Cancer Types. Cell Rep. 2018:23:213-226.e3.

38. Hardie DG. AMP-activated protein kinase—an energy sensor that regulates all aspects of cell function. Genes Dev. 2011;25:1895-908 Cold Spring Harbor Lab.

39. Shackelford DB, Shaw RJ. The LKB1-AMPK pathway: metabolism and growth control in tumour suppression. Nat Rev Cancer. 2009;9:563 Nature Publishing Group.

40. Faubert B, Boily G, Izreig S, Griss T, Samborska B, Dong Z, et al. AMPK is a negative regulator of the Warburg effect and suppresses tumor growth in vivo. Cell Metab. 2013;17:113-24 Elsevier.

41. Luo Z, Zang M, Guo W. AMPK as a metabolic tumor suppressor: control of metabolism and cell growth. Futur Oncol. 2010;6:457-70 Future Medicine.

42. Alessi DR, Sakamoto K, Bayascas JR. LKB1-dependent signaling pathways. Annu Rev Biochem. 2006;75:137-63 Annual Reviews.

43. Gurumurthy S, Hezel AF, Berger JH, Bosenberg MW, Bardeesy N. LKB1 deficiency sensitizes mice to carcinogen-induced tumorigenesis. Cancer Res. 2008;68:55-63 AACR

44. Zhang Y, Ng PK, Kucherlapati M, Mills GB, Kwiatkowski DJ, Creighton CJ. A pan-Cancer Proteogenomic atlas of PI3K/AKT/mTOR pathway alterations. Cancer Cell. 2017;31:820-32.

45. Shi WY, Xiao D, Wang L, Dong LH, Yan ZX, Shen ZX, et al. Therapeutic metformin/AMPK activation blocked lymphoma cell growth via inhibition of mTOR pathway and induction of autophagy. Cell Death Dis. 2012;3:e275 Nature Publishing Group.

46. Phoenix KN, Vumbaca F, Claffey KP. Therapeutic metformin/AMPK activation promotes the angiogenic phenotype in the ERa negative MDA-MB-435 breast cancer model. Breast Cancer Res Treat. 2009;113:101-11 Springer.

47. Laderoute KR, Amin K, Calaoagan JM, Knapp M, Le T, Orduna J, et al. 5'AMP-activated protein kinase (AMPK) is induced by low-oxygen and glucose deprivation conditions found in solid-tumor microenvironments. Mol Cell Biol Am Soc Microbiol. 2006;26:5336-47.

48. Kato K, Ogura T, Kishimoto A, Minegishi Y, Nakajima N, Miyazaki M, et al. Critical roles of AMP-activated protein kinase in constitutive tolerance of cancer cells to nutrient deprivation and tumor formation. Oncogene. 2002; 21:6082 Nature Publishing Group.

49. Frigo DE, Howe MK, Wittmann BM, Brunner AM, Cushman I, Wang Q, et al. CaM kinase kinase $\beta$-mediated activation of the growth regulatory kinase AMPK is required for androgen-dependent migration of prostate cancer cells. Cancer Res. 2011;71:528-37 AACR.

50. Park HU, Suy S, Danner M, Dailey V, Zhang Y, Li H, et al. AMP-activated protein kinase promotes human prostate cancer cell growth and survival. Mol Cancer Ther. 2009:8:733-41 AACR.

51. Wan L, Xu K, Wei Y, Zhang J, Han T, Fry C, et al. Phosphorylation of EZH2 by AMPK suppresses PRC2 methyltransferase activity and oncogenic function. Mol Cell. 2018;69:279-91 Elsevier. 
52. Jin DH, Kim Y, Bin LB, Han J, Kim HK, Shim YM, et al. Metformin induces cell cycle arrest at the G1 phase through E2F8 suppression in lung cancer cells. Oncotarget. 2017;8:101509 Impact Journals, LLC.

53. Yung MMH, Chan DW, Liu WWS, Yao K-M, Ngan HY-S. Activation of AMPK inhibits cervical cancer cell growth through AKT/FOXO3a/FOXM1 signaling cascade. BMC Cancer. 2013;13:327 BioMed Central.

54. Lee JH, Lee H-J, Sim DY, Jung JH, Kim KR, Kim S-H. Apoptotic effect of lambertianic acid through AMPK/FOXM1 signaling in MDA-MB231 breast cancer cells. Phyther Res. 2018;32:1755-63 Wiley Online Library.

55. He S, Zhao Z, Wang Y, Zhao J, Wang L, Hou F, et al. Reduced expression of SMAD4 in gliomas correlates with progression and survival of patients. J Exp Clin Cancer Res. 2011;30:70 BioMed Central.

56. Wang L-H, Kim S-H, Lee JH, Choi Y-L, Kim YC, Park TS, et al. Inactivation of SMAD4 tumor suppressor gene during gastric carcinoma progression. Clin cancer Res. 2007;13:102-10 AACR.

57. Takagi Y, Kohmura H, Futamura M, Kida H, Tanemura H, Shimokawa K, et al. Somatic alterations of the DPC4 gene in human colorectal cancers in vivo. Gastroenterology. 1996;111:1369-72 Elsevier.

58. Bartsch D, Hahn SA, Danichevski KD, Ramaswamy A, Bastian D, Galehdari H, et al. Mutations of the DPC4/Smad4 gene in neuroendocrine pancreatic tumors. Oncogene. 1999;18:2367 Nature Publishing Group.

59. Schwarte-Waldhoff I, Volpert OV, Bouck NP, Sipos B, Hahn SA, Klein-Scory S, et al. Smad4/DPC4-mediated tumor suppression through suppression of angiogenesis. Proc Natl Acad Sci. 2000;97:9624-9 National Acad Sciences.

\section{Publisher's Note}

Springer Nature remains neutral with regard to jurisdictional claims in published maps and institutional affiliations.

Ready to submit your research? Choose BMC and benefit from:

- fast, convenient online submission

- thorough peer review by experienced researchers in your field

- rapid publication on acceptance

- support for research data, including large and complex data types

- gold Open Access which fosters wider collaboration and increased citations

- maximum visibility for your research: over $100 \mathrm{M}$ website views per year

At $\mathrm{BMC}$, research is always in progress.

Learn more biomedcentral.com/submissions 